\title{
Is Geometric Frustration-Induced Disorder a Recipe for High Ionic Conductivity?
}

Andre Düvel ${ }^{1,5^{*}}$, Paul Heitjans ${ }^{1}$, Pavel Fedorov ${ }^{2}$, Gudrun Scholz ${ }^{3}$, Giannantonio Cibin ${ }^{4}$, Alan V. Chadwick ${ }^{5}$, David M. Pickup ${ }^{5}$, Silvia Ramos ${ }^{5}$, Lewis W.L. Sayle ${ }^{5}$, Emma K. Sayle $^{5}$, Thi X.T. Sayle ${ }^{5}$, Dean C. Sayle ${ }^{5 *}$

${ }^{1}$ Institute of Physical Chemistry and Electrochemistry, Gottfried Wilhelm Leibniz University Hannover, Callinstrasse 3a, D-30167 Hannover, Germany

${ }^{2}$ General Physics Institute of Russian Academy of Science, Moscow, Russia

${ }^{3}$ Department of Chemistry, Humboldt-Universität zu Berlin, Brook-Taylor-Str. 2, D-12489, Berlin, Germany

${ }^{4}$ Diamond Light Source, Harwell Science and Innovation Campus, Didcot, Oxfordshire, OX11 ODE, UK

${ }^{5}$ School of Physical Sciences, University of Kent, Canterbury, Kent CT2 7NH

* d.c.sayle@kent.ac.uk, a.duvel@kent.ac.uk

\section{ABSTRACT}

Ionic conductivity is ubiquitous to many industrially important applications such as fuel cells, batteries, sensors and catalysis. Tunable conductivity in these systems is therefore key to their commercial viability. Here, we show that geometric frustration can be exploited as a vehicle for conductivity tuning. In particular, we imposed geometric frustration upon a prototypical system, $\mathrm{CaF}_{2}$, by ball milling it with $\mathrm{BaF}_{2}$, to create nanostructured $\mathrm{Ba}_{1-x} \mathrm{Ca}_{x} \mathrm{~F}_{2}$ solid solutions and increased its ionic conductivity by over 5 orders of magnitude. By mirroring each experiment with MD simulation, including 'simulating synthesis', we reveal that geometric frustration confers, on a system at ambient temperature, structural and dynamical attributes that are typically associated with heating a material above its superionic transition temperature. These include: structural disorder, excess volume, pseudo vacancy arrays and collective transport mechanisms; we show that the excess volume correlates with ionic conductivity for the $\mathrm{Ba}_{1-x} \mathrm{Ca}_{x} \mathrm{~F}_{2}$ system. We also present evidence that geometric frustration-induced conductivity is a general phenomenon, which may help explain the high ionic conductivity in doped fluorite-structured oxides such as ceria and zirconia, with application for solid oxide fuel cells. A review on geometric frustration [Nature 2015, 512, 303] remarks that 'classical crystallography is inadequate to describe systems with correlated disorder, but that geometric frustration has clear crystallographic signatures'. Here, we identify two possible crystallographic signatures: excess volume and correlated 'snake-like' ionic transport; the latter infers correlated disorder. In particular, as one ion in the chain moves, all the other (correlated) ions in the chain move simultaneously. Critically, our simulations reveal snake-like chains, over $40 \AA$ in length, which indicates long-range correlation in our disordered systems. Similarly, collective transport in glassy materials is well documented [for example, J. Chem. Phys. 2013, 138, 12A538]. Possible crystallographic nomenclatures, to be used to describe long-range order in disordered systems, may include, for example, the shape, length, branching of the 'snake' arrays. Such 
characterizations may ultimately provide insight and differences between long-range order in disordered, amorphous or liquid states, and processes such as ionic conductivity, melting and crystallization. 


\section{Introduction}

Ionic conductivity is central to the power associated with a fuel cell ${ }^{(1,2)}$ or battery ${ }^{(3-5)}$. Accordingly, considerable and sustained efforts have focused on elucidating the mechanisms underpinning ionic transport to open new paths for the synthesis of new materials with tunable conductivities. ${ }^{(6-13)}$

One method for conductivity tuning is by interfacing two ionic conductors together. A prominent case was reported by Sata et al. ${ }^{(11)}$ who observed fluoride ion conductivities in layered $\mathrm{BaF}_{2}-\mathrm{CaF}_{2}$ composites, prepared by molecular beam epitaxy (MBE), several orders of magnitude larger than the parent binary fluorides. ${ }^{(11)}$ The authors found that the conductivities were only enhanced parallel (not perpendicular) to the layers. This phenomenon was attributed to space charge effects, which manifest in some of the fluoride ions, comprising the BaF2-layer, moving into interstitial positions in the $\mathrm{CaF}_{2}$-layer. In accord with this model, the ionic conductivity was found to increase with decreasing layer thickness. ${ }^{(11,14)}$

More recently, ionic conductivities, even higher than those reported by Sata et al. ${ }^{(11)}$ were measured for $\mathrm{BaF}_{2}-\mathrm{CaF}_{2}$ composites prepared by high-energy ball milling. ${ }^{(15,16)}$ Metastable $\mathrm{Ba}_{1-x} \mathrm{Ca}_{x} \mathrm{~F}_{2}{ }^{(17-19)}$, as well as stable $\mathrm{Ca}_{1-x} \mathrm{Sr}_{x} \mathrm{~F}_{2}{ }^{(20,21)}$, and $\mathrm{Pb}_{1-x} \mathrm{Cd}_{x} \mathrm{~F}_{2}{ }^{(22-24)}$ solid solutions, crystallizing into the fluorite structure, have also been shown to exhibit increased ionic conductivities compared to the binary parents. Specifically, fluoride ion conductivity maxima and activation energy minima, were found at intermediate compositions, $x .^{(19,20,22-24)}$ The enhanced ionic conductivity in $\mathrm{Pb}_{1-x} \mathrm{Cd}_{x} \mathrm{~F}_{2}$ was attributed to a disordered (pseudo molten) fluoride ion sublattice. ${ }^{(23,24)}$ In addition, the superionic transition temperature of $\mathrm{Pb}_{1-x} \mathrm{Cd}_{x} \mathrm{~F}_{2}$ was found to be lower than observed for the parent material, $\mathrm{PbF}_{2}{ }^{\text {(24) }}$

Here, we characterize the mechanism underpinning ionic conductivity in a prototypical nanostructured system: $\mathrm{Ba}_{1-x} \mathrm{Ca}_{x} \mathrm{~F}_{2}$. To achieve this goal, we mirror each experiment (both synthesis and property measurement) using Molecular Dynamics (MD) simulation. The direct correlation between each experiment and simulation enables additional quantitative insight.

Full atom-level models of nanostructured $\mathrm{Ba}_{1-x} \mathrm{Ca}_{x} \mathrm{~F}_{2}$ are generated by simulating the crystallization of the nanomaterial. The models are then used to mirror each experimental measurement: MD simulation to calculate the ionic conductivity and activation energy barriers measured using impedance spectroscopy; graphical techniques to visualize the structure and local disorder measured using XRD, NMR and EXAFS; calculated Connolly surfaces to determine lattice expansion measured using XRD; animations to observe the fastest moving ions and witness intuitively the structural features that facilitate fast ion conduction directly and associated transport 
mechanisms measured using NMR. Mirroring experiment both validates the simulations and instills confidence in using simulation to ascertain features that are not visible, or difficult to measure, experimentally.

\section{METHODS}

\section{Experiment}

$\mathrm{Ba}_{1-x} \mathrm{Ca}_{x} \mathrm{~F}_{2}$ samples were prepared by high-energy ball milling mixtures of $\mathrm{BaF}_{2}$ and $\mathrm{CaF}_{2}$ in a planetary ball mill. They were investigated using XRPD, impedance spectroscopy, ${ }^{19} \mathrm{~F}$ nuclear magnetic resonance (NMR) and extended X-ray absorption fine structure (EXAFS) spectroscopy. Full details of the syntheses and measurements are given in supporting information.

\section{Simulation}

Atom-level models of $\mathrm{Ba}_{1-x} \mathrm{Ca}_{x} \mathrm{~F}_{2}(x=0.00,0.25,0.50,0.75,1.00)$ nanoparticles, together with $\mathrm{BaF}_{2} / / \mathrm{CaF}_{2}$ interfaces, were generated using MD simulation and used to calculate transport mechanisms. The nanoparticles, and a segment of the $\mathrm{BaF}_{2} / / \mathrm{CaF}_{2}$ interface system, are shown in fig. 1. Full details, pertaining to the generation of the models, are presented in supporting information (figs. S1, S2 and S3).

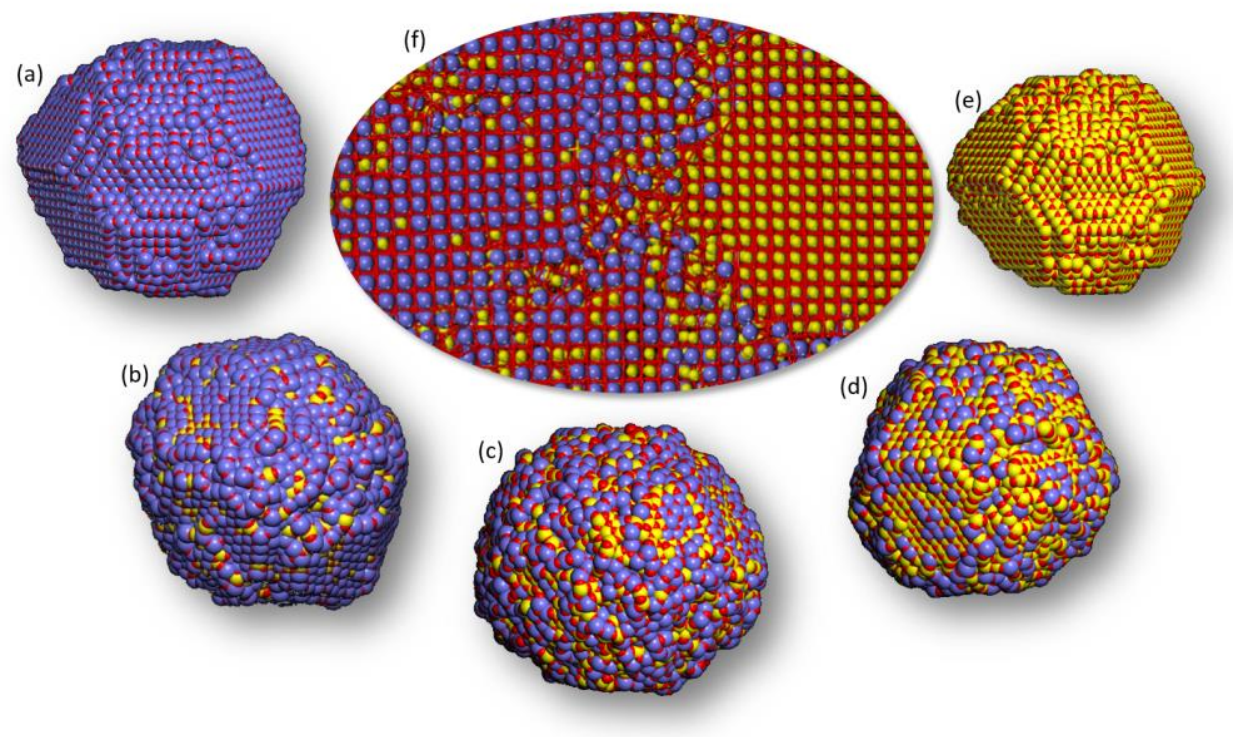

Figure 1 Model structures for (a-e) nanoparticle solid solutions and (f) BaF2//CaF $\mathrm{F}_{2}$ interfacial system. (a) BaF2 parent, (b) $\mathrm{Ba}_{0.75} \mathrm{Ca}_{0.25} \mathrm{~F}_{2}$, (c) $\mathrm{Ba}_{0.50} \mathrm{Ca}_{0.50} \mathrm{~F}_{2}$, (d) $\mathrm{Ba}_{0.25} \mathrm{Ca}_{0.75} \mathrm{~F}_{2}$, (e) $\mathrm{CaF}_{2}$ parent. Barium is colored blue, calcium is yellow and fluoride is red. 


\section{Results}

\section{Ionic conductivity and activation energy barriers}

Ionic conductivities of $\mathrm{Ba}_{1-x} \mathrm{Ca}_{x} \mathrm{~F}_{2}$ samples, measured as a function of composition $x$, are shown in fig 2(a) together with values calculated from MD simulations in fig 2(b). The figures reveal a broad conductivity maximum and activation energy, $E_{\text {act, }}$ minimum for nanoparticle compositions around $x=0.50$. Experimentally, the conductivity of $\mathrm{CaF}_{2}$ at $373 \mathrm{~K}$ is seen to increase by 5 orders of magnitude when doped with $50 \% \mathrm{Ba}$ at $373 \mathrm{~K}$. For $x>0.7$ the conductivity drops sharply and is associated with a sharp increase in $E_{\text {act. }}$ Conversely, the $\mathrm{BaF}_{2} / / \mathrm{CaF}_{2}$ interface system, fig 2(b), shows comparatively lower calculated conductivities and higher $E_{\text {act. }}$ We now examine the structures, at the atom level, to ascertain the origin of the conductivity maxima.
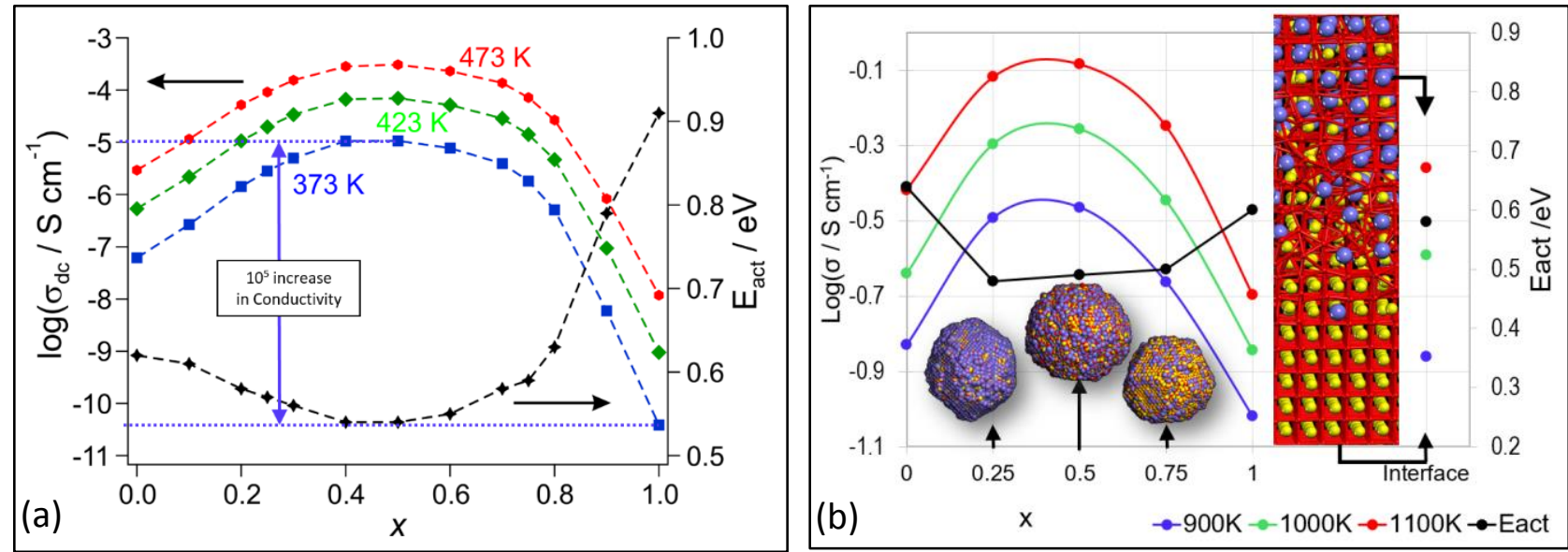

Figure 2 (a) Measured and (b) calculated F ion conductivities and associated activation energy barriers, $E_{a c t,}$ for $B a_{1}$ ${ }_{x} \mathrm{Ca}_{x} F_{2}$ nanoparticle solid solutions, as a function of $x$. The atom level models of the nanoparticle solid solutions, and $\mathrm{BaF}_{2} / / \mathrm{CaF}_{2}$ interface are also shown in (b); Ba is colored blue, Ca is yellow and $\mathrm{F}$ is red. 


\section{Structural Disorder}

Analysis of the real material, reveals that high ionic conductivity correlates with high structural disorder: EXAFS spectra are broadest (most disordered) at $x=0.50$, fig 3; NMR shows maximum disorder at $x=0.50$, fig. S15; the atomistic models are most disordered for $x=0.50$, figs 4,56 and 58 . We note that the structural disorder of the model $\mathrm{Ba}_{1-\times \mathrm{C}} \mathrm{C}_{\times} \mathrm{F}_{2}$ solid solutions at low (0 K) temperature are similar to those of the parent materials, but at high (1000 K) temperature, fig 4.

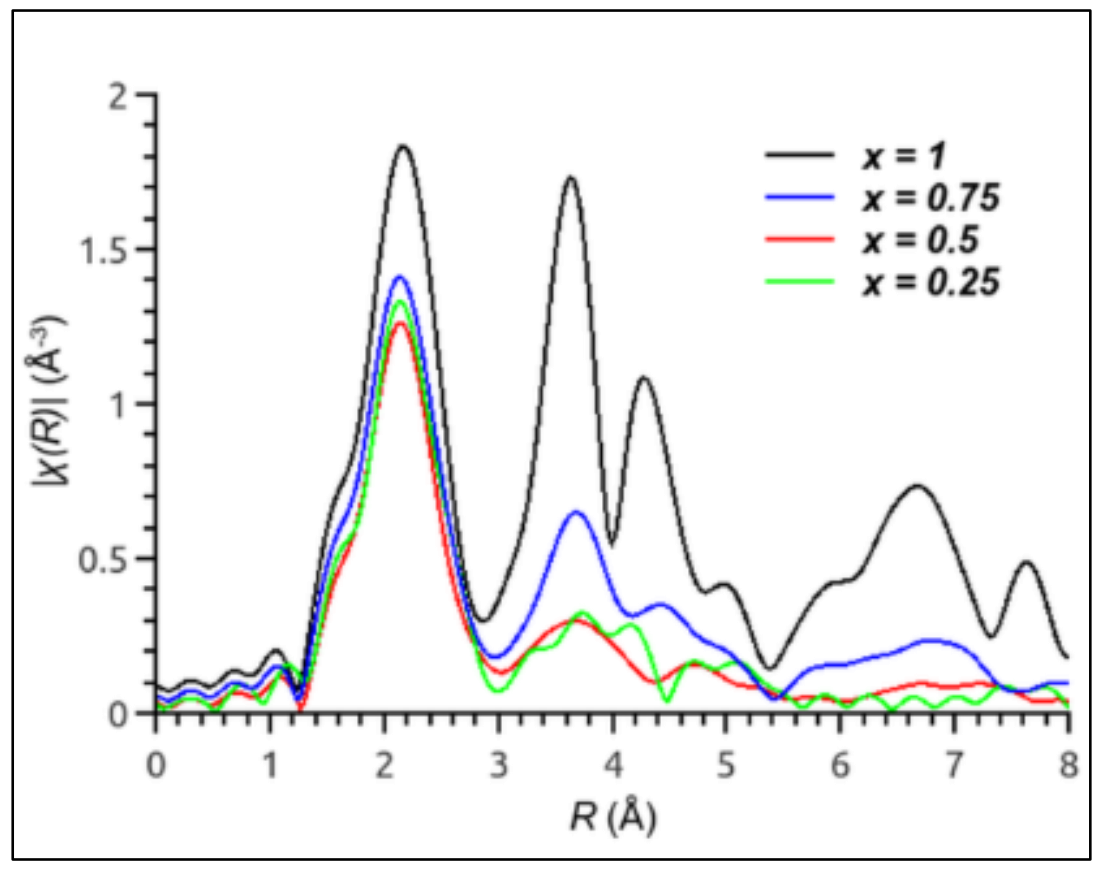

Figure 3 FT of the normalized Ca K edge EXAFS of $\mathrm{Ba}_{1-x} \mathrm{Ca}_{\times} \mathrm{F}_{2}$.

\section{Excess Volume}

The excess (thermodynamic) volumes of the model $\mathrm{Ba}_{1-\mathrm{x}} \mathrm{Ca}_{x} \mathrm{~F}_{2}$ nanoparticle solid solutions, calculated as a function of $\mathrm{x}$, are shown in fig 4 . The figure reveals that the excess volume increases as $\mathrm{BaF}_{2}$ is gradually doped with $\mathrm{Ca}$ and reaches a maximum at $x=0.50\left(\mathrm{Ba}_{0.50} \mathrm{Ca}_{0.50} \mathrm{~F}_{2}\right)$. Excess volume is calculated as the difference between the volume of the nanoparticle solid solutions and the volumes of the constituent parent materials, $\mathrm{BaF}_{2}$ and $\mathrm{CaF}_{2}$, in their respective perfect lattices. The shape of the curve in fig 4 supports the assumption of an 'atom-level scaffold', i.e. the large Ba ions hold open the lattice until their number becomes insufficient to do so.

In accord with our simulations, experimentally we find that the lattice parameters of the $\mathrm{Ba}_{1-\mathrm{x}} \mathrm{Ca}_{x} \mathrm{~F}_{2}$ nanoparticle solid solutions exhibit positive deviations from Vegard behavior, with a maximum around $x=0.3-0.4$; fig. S14 
shows how the lattice parameter changes as a function of $x$ and fig. S18 reveals local cation-fluoride distances, as a function of $x$, estimated using ${ }^{19} \mathrm{~F}$ MAS NMR. We note that the XRPD patterns of the $\mathrm{Ba}_{1-x} \mathrm{Ca}_{x} \mathrm{~F}_{2}$ solid solutions, fig. S13, show single phase compounds, except for the samples with $x \leq 0.25$, which also contain small amounts of orthorhombic $\mathrm{Ba}_{1-x} \mathrm{Ca}_{x} \mathrm{~F}_{2}$ (see $\mathrm{SI}$ for more information). ${ }^{19} \mathrm{~F}$ MAS NMR spectra of the samples (fig. S15) show atomlevel cation intermixing, confirming the formation of solid solutions.

We note that maximum excess volume, fig 4 , correlates with maximum conductivity and minimum activation energy, fig 2. On a related note, simulation reveals that $\mathrm{CeO}_{2}, \mathrm{CaF}_{2}$ and $\mathrm{PbF}_{2}$, under tensile strain, exhibit reduced activation energy barriers for ionic transport ${ }^{(25-27)}$. Since tensile strain increases the space in which the ions have to move, we propose that the excess volume, associated with our systems, provides additional space for ionic mobility. This also explains the reduced superionic transition temperature ${ }^{(27)}$ observed for e.g. $\mathrm{Pb}_{1-x} \mathrm{Cd}_{x} \mathrm{~F}_{2}{ }^{(24)}(\mathrm{see}$ also SI). Moreover, doping is a technologically easy method for imposing 'tensile strain' upon a system - perhaps akin to an 'atom-level scaffold'. 


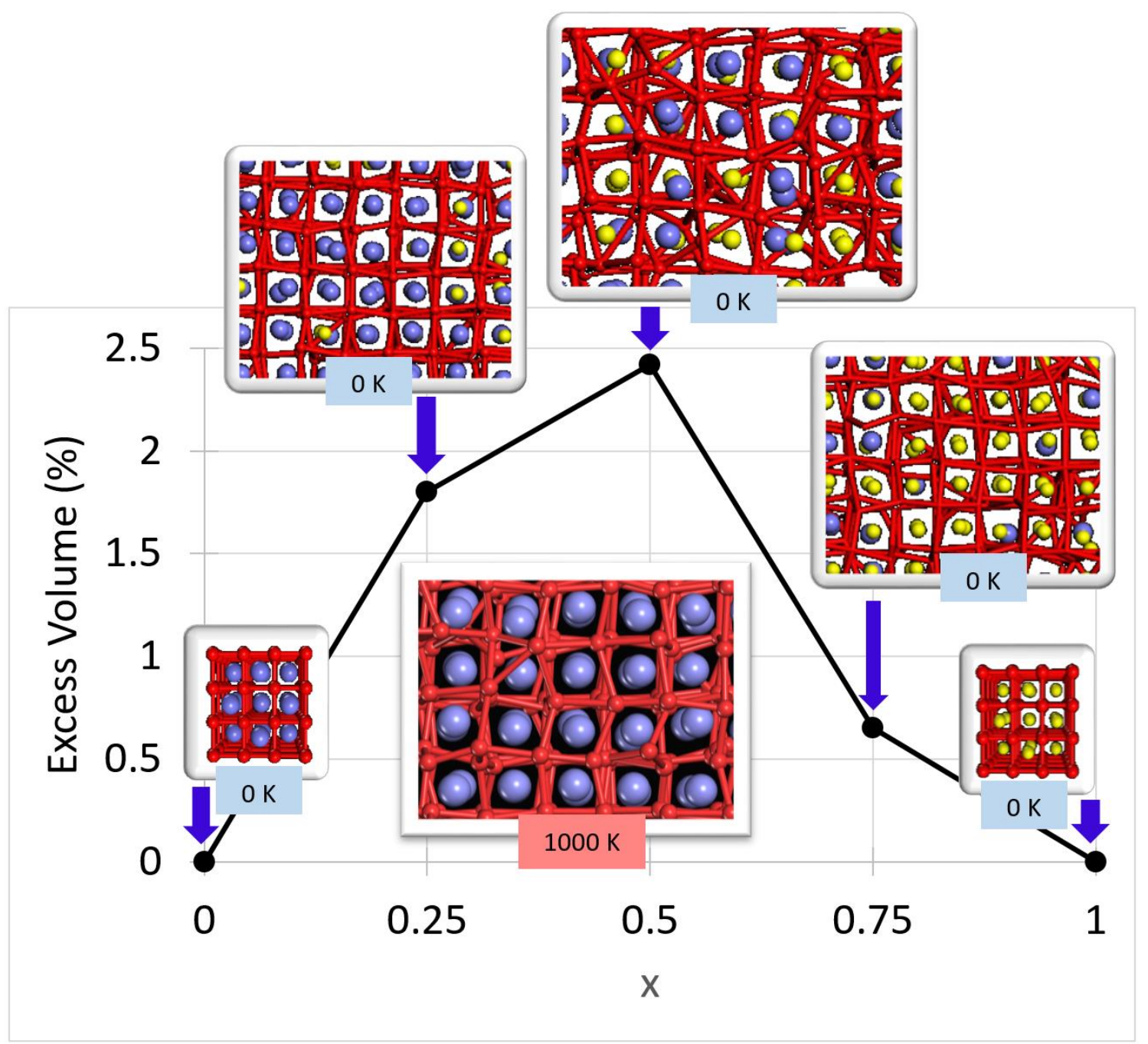

Figure 4 Excess volumes of the model $\mathrm{Ba}_{1-x} \mathrm{Ca}_{x} \mathrm{~F}_{2}$ nanoparticles, calculated as a function of composition, $x$. We note that excess volume correlates with structural disorder; segments of the structure are shown for each composition. The structure of pure $\mathrm{BaF}_{2}$ at $1000 \mathrm{~K}$ (a snapshot in time) is also shown and reveals similar disorder to the doped $\left(B a_{1-x} C a_{x} F_{2}\right)$ systems at zero Kelvin, indicating that doping can induce similar structural changes, and hence mobility, that are typically associated with heating the system by $1000 \mathrm{~K}$.

\section{Ionic Transport}

The fastest moving fluoride ions are in domains of highest structural disorder: static ${ }^{19} \mathrm{~F}$ NMR spectra of the system with highest disorder, $\mathrm{Ba}_{0.50} \mathrm{Ca}_{0.50} \mathrm{~F}_{2}$, shows a motional narrowing of the NMR trace as a function of temperature indicating highly mobile ions at $T>453 \mathrm{~K}$ for this system, fig. 5 . Conversely, the $\mathrm{NMR}$ trace of the $\mathrm{BaF}_{2}-\mathrm{CaF}_{2}$ composite, at $T>453 \mathrm{~K}$, comprises two shoulders that are indicative of slow moving fluoride ions in the almost 
pure $\mathrm{BaF}_{2}$ and $\mathrm{CaF}_{2}$ domains in addition to a sharp central peak (highly mobile ions). Further details are provided in supporting information.

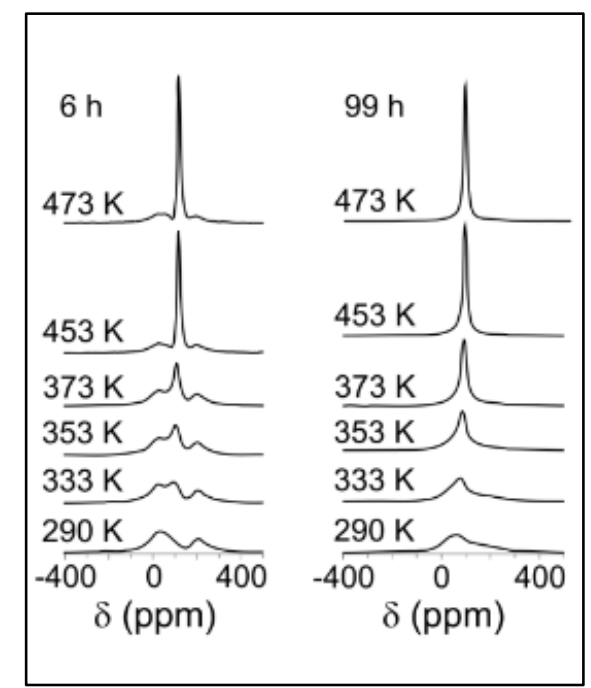

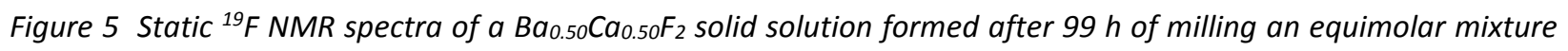
of $\mathrm{BaF}_{2}$ and $\mathrm{CaF}_{2}$ employing a $\mathrm{ZrO}_{2}$ milling vial set (right trace) and a BaF$-\mathrm{CaF}_{2}$ composite formed after only $6 \mathrm{~h}$ of milling a similar mixture in the same milling vial set (left trace).

\section{Transport Mechanism}

Animations of the MD simulations, available in supporting information, reveal intuitively that the fastest moving $\mathrm{F}$ ions are located in domains of high disorder, fig 6(a), in accord with our experiments. Conversely, F ions, located in regions of atomically sharp $\mathrm{BaF}_{2} / / \mathrm{CaF}_{2}$ interface regions, fig 6(b), are much less mobile.

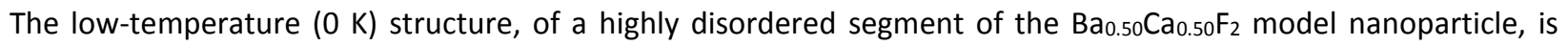
shown in fig 6(c). All the F ions are displaced far from their perfect lattice sites leaving an array of pseudo vacancies (white squares). The animations reveal that $\mathrm{F}$ ion transport proceeds via a collective mechanism - several $\mathrm{F}$ ions move simultaneously through the material in a 'snake-like' movement, fig 6(c), fig S12. F ions move through the lattice along <100> and occasionally <110> or <111> directions. We also observe collective 'snake-like' movement of $\mathrm{F}$ ions in the $\mathrm{BaF}_{2}$ and $\mathrm{CaF}_{2}$ end members albeit with a much reduced jump frequency compared to the nanoparticle solid solutions.

Inspection of fig $6(\mathrm{a})$ reveals considerable oscillatory motion of the $\mathrm{F}$ ions - analogous to thermal ellipsoids. The largest ellipsoids are seen for $\mathrm{F}$ ions in mixed $(\mathrm{Ba}, \mathrm{Ca})$ cationic environments. We propose that the conflicting interatomic interactions with the neighbouring Ba and Ca cations results in geometric frustration. ${ }^{(28)}$ This increases 
the packing space (excess volume) required to accommodate all the ions and consequently provides additional space for $\mathrm{F}$ ions to move.

We postulate that there will be a distribution of local energy minima for fluoride ions that exist within ellipsoids, which will reduce the activation energy barriers associated with $\mathrm{F}$ ions moving between sites. The overlap between neighboring ellipsoids indicates facile collective motion of the ions; collective ion transport was reported for several superionic conductors ${ }^{(29-33)}$ and ascribed to a dynamical change of local (frustrated) bonding. ${ }^{(34-36)}$
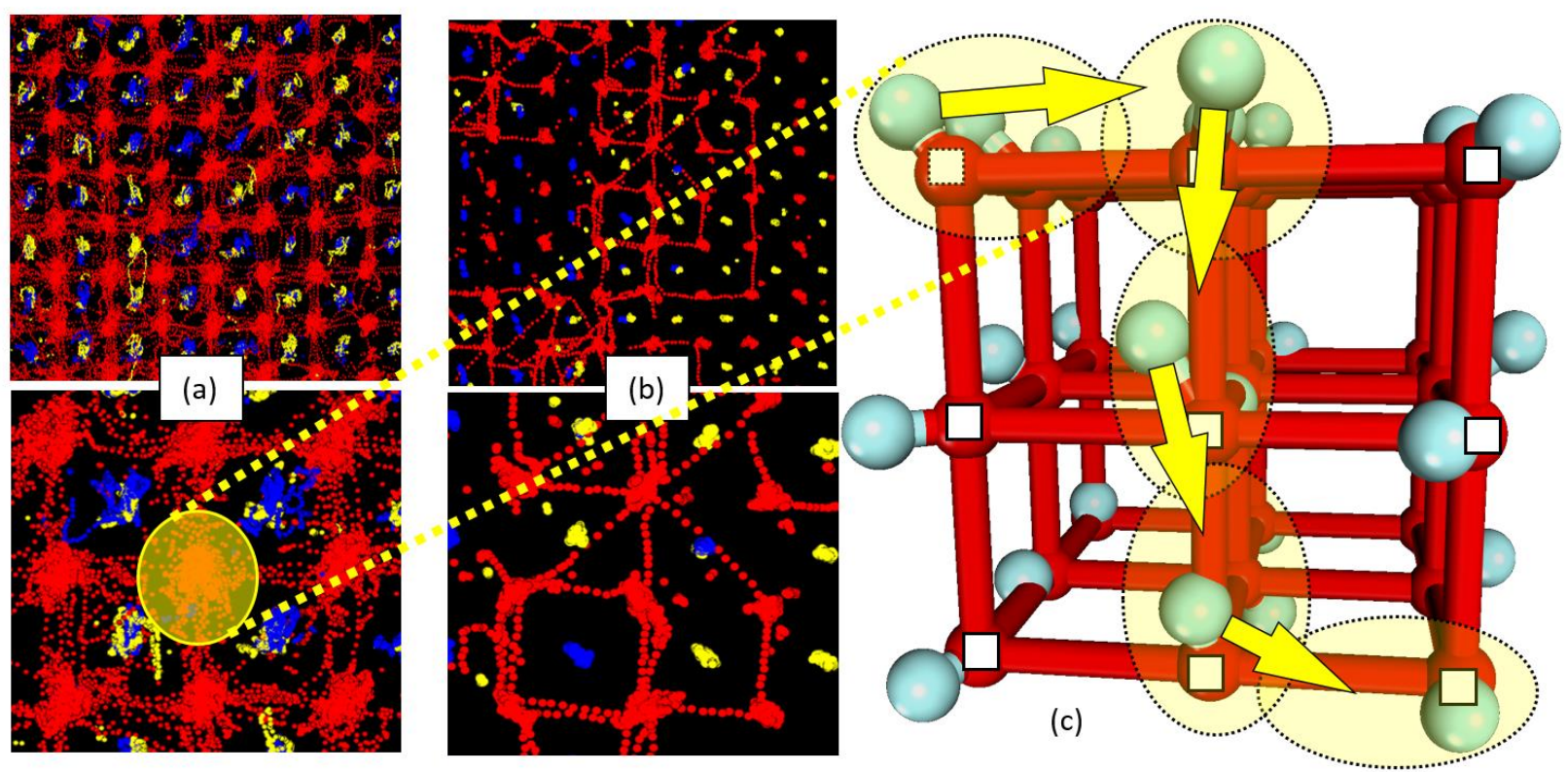

Fig $6 \mathrm{~F}$ ion trajectories mapped during MD simulation (a) Ba0.50 $\mathrm{Ca}_{0.50} \mathrm{~F}_{2}$ nanoparticle, (b) $\mathrm{BaF}_{2} / / \mathrm{CaF}_{2}$ interface. (a) and (b) show superimposed snapshots of atom positions taken over $25 \mathrm{ps}$ of MD at $900 \mathrm{~K}$. (c) $0 \mathrm{~K}$ image of $\mathrm{F}$ atom positions (blue) of part of the $\mathrm{Ba}_{0.50} \mathrm{Ca}_{0.50} \mathrm{~F}_{2}$ model structure superimposed on the perfect lattice (red) showing the pseudo vacancies; arrows depict the simultaneous movement of $F$ ions, residing in the light-yellow ellipsoids, in a 'snake-like' mechanism. 


\section{Discussion}

Computer modelling has reached a maturity whereby both synthesis and property measurement can be mirrored directly with computer simulation. Here, we demonstrate and then exploit this capability to elucidate the origin of the 5 orders of magnitude increase in ionic conductivity when nanostructured $\mathrm{CaF}_{2}$ is doped with $\mathrm{Ba}$.

\section{Synthesis}

- Experiment Ball-mill induced partial amorphization of nanoparticle precursors followed by crystallization.

- Simulation Intimate mixing of amorphous $\mathrm{BaF}_{2}$ and $\mathrm{CaF}_{2}$ precursors followed by (direct) crystallization using MD simulation; nanoparticles generated by direct crystallization of a fluorite-structured nanoparticle followed by doping of cation positions.

\section{$1 \quad$ Ionic Conductivity and Activation Energy Barriers}

- Experiment: Maximum ionic conductivity and minimum activation energy barriers, at $x \approx 0.50$, fig 2(a) (impedance spectroscopy).

-Simulation: Maximum ionic conductivity and minimum activation energy at $x \approx 0.50$, fig 2 (b) (MD simulation).

\section{$2 \quad$ Structural Disorder}

-Experiment: $\quad$ Maximum disorder around $x=0.50\left({ }^{19} \mathrm{~F}\right.$ MAS NMR and EXAFS, figs 3 and S15).

- Simulation: Maximum disorder around $x=0.50$, fig. 4, figs. S6 and S8; geometrically frustrated fluoride ions, fig $6(\mathrm{c})$.

3

Excess Volume

- Experiment: Maximum excess (increased) lattice parameter around $x=0.35$, fig S14 (XRD).

-Simulation: Maximum excess volume around $x=0.50$ (calculated Connolly surfaces, not shown).

\section{$4 \quad$ Transport Mechanism}

- Experiment: Highest $\mathrm{F}$ ion mobility in cation intermixed regions ( ${ }^{19} \mathrm{~F} N \mathrm{NM}$, fig 5).

- Simulation: Fastest moving $\mathrm{F}$ ions in intermixed regions; collective $\mathrm{F}$ ion mobility (animations of the MD simulations, animations $\mathrm{SI}$ ). 
Experiment and simulation reveal a direct correlation between conductivity and disorder; maximum ionic conductivity coincides with both maximum disorder and maximum excess volume and occurs at $x \approx 0.50$. This can be explained when one considers that mixing large $\left(\mathrm{Ba}^{2+}=1.42 \AA^{(37)}\right)$ and small $\left(\mathrm{Ca}^{2+}=1.12 \AA^{(37)}\right)$ cations, to form a solid solution, causes geometric frustration. In particular, the conflicting interatomic interactions prevent the component $\mathrm{Ba}\left(\mathrm{F}_{2}\right)$ and $\mathrm{Ca}\left(\mathrm{F}_{2}\right)$ 'building blocks' from packing together coherently. This results in a (frustrationinduced) disordered structure that takes up more space than the component building blocks. Such excess volume provides additional space for the $\mathrm{F}$ ions to move in a correlated fashion through the lattice. We postulate that it is the combination of all these factors that give rise to the $10^{5}$ increase in conductivity. Specifically:

-Structural disorder

- Excess volume

-Pseudo vacancy array / geometrically frustrated fluoride ions

- Collective transport mechanism

Moreover, all these structural features are exhibited by fluorite-structured materials when heated above their transition temperature for superionic conduction. In particular, they have disordered sublattices of the mobile ion species $^{(6)}$; they show a discontinuity in their lattice parameter (excess volume)(38,39); they comprise pseudo vacancies $^{(6)}$ and are characterized by a collective transport mechanism ${ }^{(29-36)}$. The latter is not yet completely understood, especially for fluorite-structured materials, but is typical for homogeneously disordered systems. ${ }^{(13,40)}$

Here, we find that geometric frustration confers all these attributes on our system, but at lower temperatures, to facilitate up to 5 orders of magnitude increase in ionic conductivity. Moreover, extrapolation of the superionic region of the Arrhenius plot for $\mathrm{BaF}_{2}$ conductivity, gives good agreement with that for $\mathrm{Ba}_{0.50} \mathrm{Ca}_{0.50} \mathrm{~F}_{2}$, see fig. S20(c). Accordingly, we postulate that inducing geometric frustration manifests as a recipe for conferring fast ion conduction upon new materials at low temperature.

The close accord between experiment and simulation both validates the simulation and proffers confidence in reliable prediction emanating from simulation. In particular, simulation provided additional insight that was difficult to ascertain experimentally. Specifically, simulation revealed that the $10^{5}$ increase in ionic conductivity, observed experimentally, is not attributed to enhanced diffusion at the nanoparticle surfaces, at $\mathrm{BaF}_{2} / / \mathrm{CaF}_{2}$ interfaces or within grain-boundary regions (see SI). We also found no evidence for the formation of space charge regions but indications (simulation and experiment, at the temperature used by Sata et al. ${ }^{(11)}$ ) for cation intermixing in the interfacial regions of $\mathrm{BaF}_{2}$ and $\mathrm{CaF}_{2}$ (see $\mathrm{SI}$ for more information), which is also expected from the phase diagram ${ }^{(18)}$. 


\section{Geometric Frustration}

When two materials are interfaced together, there is geometric frustration between maximising favourable interatomic interactions across the interface and accommodating the difference in lattice parameters of the two materials. Such frustration leads to localised strain, which changes the properties of the component materials, and can be exploited in, for example, supported catalysis, microelectronics, composite materials (strength); a branch of mathematics - lattice theory - is devoted to characterising relationships between lattices and is well developed. ${ }^{(41)}$ Such understanding has aided the design of new materials with controllable properties. Heterointerface systems may be considered as a special two-dimensional case of a more general, three-dimensional, geometrical frustration, the emerging electronic effects of which are currently widely explored and exploited in frustrated magnetism.

A recent review by Keen and Goodwin ${ }^{(28)}$ states that 'It is remarkable that, for all its indisputable successes, the language of classical crystallography still cannot properly describe the structure of water ice.' They provide evidence that correlated disorder (e.g. as a consequence of geometric frustration) has clear 'crystallographic signatures'. Here, we attempt to unravel some of the 'crystallographic signatures' of geometric frustration.

In particular, we induce geometric frustration in $\mathrm{CaF}_{2}$ by doping with $\mathrm{Ba}$, which imposes considerable structural complexity (correlated disorder) upon the fluorite lattice because of the conflicting interatomic forces associated with mixing small $(\mathrm{Ca})$ and large $(\mathrm{Ba})$ ions together; the ionic conductivity of $\mathrm{CaF}_{2}$ is increased by 5 orders of magnitude. However, the structural complexity makes characterisation difficult. Locally, the structure appears heavily disordered yet globally, the fluorite structure is retained. It is difficult to map the local, 'disordered', structure onto any established crystallographic description and therefore, in accord with the recommendations of Keen and Goodwin, ${ }^{(28)}$ we look for crystallographic signatures of geometric frustration as a first step in its quantification.

We identify two signatures of geometric frustration: excess volume (the difference in volume between the frustrated $\mathrm{Ba}_{1-\mathrm{x}} \mathrm{Ca}_{x} \mathrm{~F}_{2}$ solid solution and the component parent materials, $\mathrm{BaF}_{2}$ and $\mathrm{CaF}_{2}$ ) and correlated ion transport, the latter infers correlated disorder. Visualisation of the MD simulation trajectories enables us to map correlated 'snake-like' transport pathways, such as fig 6(c) and S12. We speculate that mapping and then comparing the correlated transport pathways of all the mobile ions might present insight into correlated disorder and provide additional crystallographic signatures of geometric frustration. Ultimately, a truly random structure is unphysical and therefore the quantification of geometric frustration might present new paths to insights into characterising complex structures and even towards amorphous systems such as glass and liquids. ${ }^{(42)}$ For example, previously, we presented evidence that the crystalline seed, which evolves spontaneously and nucleates the 
crystallisation of a ceria nanoparticle from a melt, is itself a liquid at the embryonic stages; signatures of structural ordering were observed to spontaneously evolve from a molten (disordered) precursor. ${ }^{(43)}$ We speculate that to facilitate the spontaneous evolution of order, the surrounding (amorphous) ions must themselves be correlated.

\section{Dynamic Frustration}

In addition to the geometrically frustrated solid solutions, our simulations also reveal correlated 'snake-like' transport in the (undoped) parent materials, $\mathrm{BaF}_{2}$ and $\mathrm{CaF}_{2}$. We postulate that at high temperatures, the parent materials can also be considered to be frustrated, fig 4. For example, thermal energy may force a particular $\mathrm{F}$ ion off its lattice site and consequently induce a force upon its neighbouring ions, causing them to also be displaced off their lattice sites. The difference between geometric and dynamic frustration is that doping (geometric frustration) can induce disorder, and hence increase ionic conductivity, at low temperatures. We note Zhang and co-workers found collective motion during melting and suggest that 'superionic crystals might be a system exhibiting incipient homogeneous melting' (see SI for a possible explanation of superionic conduction in fluorite structured materials). ${ }^{(44)}$

\section{Crystallography of Disordered Systems}

Crystallography maps relationships between atoms as a function of distance. For materials with high crystallinity, the correlations extend over long distances. Conversely, for disordered or amorphous materials, we are only able to characterize short-range correlations, such as nearest neighbor, and presume that they have no long-range order. This is because no method for mapping long-range order in a disordered system is currently available. Conversely, our simulations reveal correlated 'snake-like' motion of ion chains. The correlated motion enables us to identify correlated ions - we observe snakes $40 \AA$ long, fig S12, which is much longer than typical (3 $\mathrm{A}$ ) nearest neighbor distances. Accordingly, we postulate that long-range order in disordered systems may be characterized by the nature of the snake arrays, such as their shape, length, branching. Moreover, Zhang et al. revealed 'stringlike cooperative motion in homogeneous melting ${ }^{\prime(44)}$ and that cooperative motion in glassy (amorphous) materials is well-established. Accordingly, we propose that crystallographic nomenclatures, used to describe long-range correlations between atoms in disordered systems, can be categorized via 'snake-like' arrays. We speculate that such representations may provide insight into the structural (long-range) differences between disordered, amorphous or liquid states and hence properties such as ionic conductivity, melting and crystallization.

Generality of geometric frustration tuned ion conduction

If our postulate, that geometric frustration can be exploited to tune the ionic conductivity, then one might expect other doped fluorite-structured ionic conductors to display similar attributes. Recent measurement in our 
laboratories revealed that, in addition to the systems mentioned in the introduction, also $\mathrm{Pb}_{1-x} \mathrm{Ca}_{x} \mathrm{~F}_{2}$ shows a clearly increased fluoride ion conductivity and decreased activation energy at intermediate compositions (ionic radii: $\left.r\left(\mathrm{~Pb}^{2+}\right)=1.29 \AA ; r\left(\mathrm{Ca}^{2+}\right)=1.12 \AA^{(37)}\right)$. We expect to observe this effect also for $\mathrm{Ba}_{1-x} \mathrm{Cd}_{x} \mathrm{~F}_{2}$ (ionic radii: $r\left(\mathrm{Ba}^{2+}\right)=1.42 \AA$; $\left.r\left(\mathrm{Cd}^{2+}\right)=1.1 \AA^{(37)}\right)$ if it can be synthesized. Clearly, if one can quantify/map geometric frustration, similar to interface systems, one can exploit its consequences.

A technologically important material, ceria, $\mathrm{CeO}_{2}$, when doped, can also exhibit increased oxygen ion conductivities with application for solid oxide fuel cells. ${ }^{(1,2)}$ Here, geometric frustration must play a key role: when $\mathrm{Ce}^{4+}$ is replaced by a different ion, geometric frustration will be induced because of the conflicting interatomic interactions. This is evidenced by the change in oxygen ion conductivity with dopant size. ${ }^{(45)}$ Aliovalent, $\mathrm{M}^{3+}$, doping leads to charge compensating oxygen vacancies, which will also frustrate the system because local interactions will change after removing an oxygen ion. Geometric frustration in these systems is also evidenced by the considerable structural disorder that the dopants and vacancies impart upon the fluorite lattice ${ }^{(46)}$. Conversely, such disorder has not, to our knowledge, been mapped to any crystallographic description. A further complication of aliovalent-doped fluorite-structured oxides is that the dopant-vacancy association energy can be high - strongly bound vacancies can reduce the conductivity because vacancies play an integral part of the transport mechanism. ${ }^{(47,48)}$ Such association again emanates from frustration. We also speculate that vacancies may 'break' or terminate the chain of correlated (oxygen) ions.

Clearly, the additional complexities of this, and similar systems, are high and even after more than 30 years of study there is no agreed consensus on absolute mechanisms. Consequently, targeted synthesis is not as well informed as one would desire. If we are able to understand and characterize geometric frustration, such understanding will aid in its exploitation. As Keen and Goodwin advocate: crystallographic signatures are the first step in this endeavor. ${ }^{(28,42)}$

\section{Design of New Ionic Conductors}

Tunable conductivity, via structural control, is desirable for the fabrication of new ionic conductors. In $\mathrm{Ba}_{1-\mathrm{x}} \mathrm{Ca}_{\mathrm{x}} \mathrm{F}_{2}$ conductors, a key question to answer is: How does the local environment of (F) ions influence the ionic conductivity?

However, this question is not trivial. This is because F transport is correlated and proceeds via a snake-like mechanism with each (F) member of the chain located in a different environment. Accordingly, one would not only have to map the different environments of each $\mathrm{F}$ member of the chain, but also the frustrated interactions between neighbouring environments. 
We propose that crystallographic mapping of the chains, might help us to answer this question in future. In particular, crystallography requires additional nomenclature to describe these complex, geometrically frustrated, structures because labeling them as 'disordered' does little to help us exploit their true potential.

\section{Conclusion}

The ionic conductivity of $\mathrm{CaF}_{2}$ can be increased by doping with $\mathrm{Ba}$ to induce geometric frustration upon the system; a $10^{5}$ increase in $\mathrm{F}$ ion conductivity was achieved. Using experimentation and simulation we find that geometric frustration increases the size of the system (excess volume) giving more space for ionic mobility. The extra space leads to a reduction in the activation energy barriers and an increase in ionic conductivity. The conductivity is tunable as a function of geometric frustration. Maximum conductivity correlates with maximum excess volume.

It is currently accepted that disordered systems have no long-range order. Here, our simulations reveal correlated transport in a disordered system. Specifically, the ions move in a snake-like motion - as one ion in the chain moves, all the other (correlated ions) in the chain move simultaneously. This infers that all the ion (positions) within the snake are correlated. Thus far we have identified snake-like chains over $40 \AA$ in length, which is much larger than short-range (nearest-neighbor) distances, which are typically up to $3 \AA$. Accordingly, our findings identify longrange order in disordered systems. The structure of the snake-like arrays may therefore provide the key to understanding differences in the long-range order in disordered, amorphous or liquid states, and processes such as ionic conductivity, melting and crystallization.

\section{Acknowledgment}

We thank Dr. Reinhard Uecker (IKZ, Berlin) for supporting us with the $0.7 \mathrm{BaF}_{2}: 0.3 \mathrm{CaF}_{2}$ quenched melt. AD is grateful for financial support by the German science foundation (DFG) in the frame of the priority program 1415 and DU1668/1-1) and by Leibniz University Hannover in the frame of "Wege in die Forschung II". EPSRC: EP/H001220.

References 
(1) Malavasi, L.; Fisher, C. A. J.; Islam, M. S. Chem. Soc. Rev. 2010, 39, 4370-4387.

(2) Orera, A.; Slater, P. R. Chem. Mater. 2010, 22, 675-690.

(3) Reddy, M. A.; Fichtner, M. J. Mater. Chem. 2011, 21, 17059-17062.

(4) Gschwind, F.; Rodriguez-Garcia, G.; Sandbeck, D. J. S.; Gross, A.; Weil, M.; Fichtner, M.; Hörmann, N. J. Fluorine Chem. 2016, 182, 76-90.

(5) Choi, N.-S.; Chen, Z.; Freunberger, S.A.; Ji, X.; Sun, Y.-K.; Amine, K.; Yushin, G.; Nazar, L.F.; Cho, J.; Bruce, P.G. Angew. Chem. Int. Edit. 2012, 51, 9994-10024.

(6) Hull, S. Rep. Prog. Phys. 2004, 67, 1233-1314.

(7) Sorokin, N.I. Russ. J. Electrochem. 2006, 7, 744-759.

(8) Goodenough, J.B. Annu. Rev. Mater. Res. 2003, 33, 91-128.

(9) Patro, L.N.; Hariharan, K. Solid State Ionics, 2013, 239, 41-49.

(10) Maier, J. Nature Materials 2005, 4, $805-815$.

(11) Sata, N.; Ebermann, K.; Eberl, K.; Maier, J. Nature 2000, 408, 946-949.

(12) Ivanov-Shitz, A.K. Crystallogr. Rep. 2007, 52, 129-140.

(13) Dyre, J. C.; Maass, P.; Roling, B.; Sidebottom, D. L. Rep. Prog. Phys. 2009, 72, 046501 (15pp).

(14) Jin-Phillipp, N. Y.; Sata, N.; Maier, J.; Scheu, C.; Hahn, K.; Kelsch, M.; Rühle, M. J. Chem. Phys. 2004, 120, 23752381.

(15) Ruprecht, B.; Wilkening, M.; Steuernagel, S.; Heitjans, P. J. Mater. Chem. 2008, 18, 5412-5416.

(16) Ruprecht, B.; Wilkening, M.; Feldhoff, A.; Steuernagel, S.; Heitjans, P. Phys. Chem. Chem. Phys. 2009, 11, 30713081.

(17) $\mathrm{Ba}_{1-x} \mathrm{Ca}_{x} \mathrm{~F}_{2}$ solid solutions do not exist continuously, at equilibrium, for all values of $x^{(18)}$ rather there are only limited solid solutions on the basis of the $\mathrm{CaF}_{2}-\mathrm{BaF}_{2}$ phase diagram. In this respect it differs from solid solutions, such as $\mathrm{Ca}_{1-x} \mathrm{Sr}_{x} \mathrm{~F}_{2}$ and $\mathrm{Cd}_{1-x} \mathrm{~Pb}_{x} \mathrm{~F}_{2}$. Mechanochemical synthesis and $\mathrm{MBE}$ are the only known methods to synthesize it. However, the prepared samples are rather stable. Storage of $\mathrm{Ba}_{0.50} \mathrm{Ca}_{0.50} \mathrm{~F}_{2}$ for two years under air showed no change observable by XRPD. However, after four years under air partly decomposition and oxidation (which was, however, also observed for other mechanically activated, nanocrystalline fluorides) was observed. Also heating the compounds with intermediate compositions $x$ at temperatures beyond $700 \mathrm{~K}$ for some hours lead to decomposition ${ }^{(19)}$. We have not observed any clear signs of decomposition due to heating of our simulated nanoparticles at temperatures of up $1200 \mathrm{~K}$, which is most likely due to the very short observation times of no more than 500 ps.

(18) Fedorov, P. P.; Buchinskaya, I. I.; Ivanovskaya, N. A.; Konovalova, V. V.; Lavrishchev, S. V.; Sobolev, B. P. Dokl. Phys. Chem. 2005, 401, 652-654.

(19) Düvel, A.; Ruprecht, B.; Heitjans, P.; Wilkening, M. J. Phys. Chem. C 2011, 115, 23784-23789.

(20) Subrahmanya Sarma, M. V.; Suryanarayana, S. V. Solid State Ionics 1990, 42, 227-232.

(21) Karimov, D. N.; Komar'kova, O. N.; Sorokin, N. I.; Bezhanov, V. A.; Chernov, S. P.; Popov, P. A.; Sobolev, B. P. 
Crystallogr. Rep. 2010, 55, 518-524.

(22) Murin, I. V.; Chernov, S. V. Inorg. Mater. 1982, 18, 149-150.

(23) Kosacki, I.; Litvinchuk, A. P.; Tarasov, J. J.; Ya Valakh, M. J. Phys.: Condens. Matter 1989, 1, 929-934.

(24) Kosacki, I. Appl. Phys. A 1989, 49, 413-424.

(25) De Souza, R. A.; Ramadan, A.; Hörner, S. Energy Environ. Sci. 2012, 5, 5445-5453.

(26) Rushton, M. J. D.; Chroneos, A. Sci. Rep. 2014, 4, 6068.

(27) Cazorla, C.; Errandonea, D. Nano Lett. 2016, 16, 3124-3129.

(28) Keen, D. A.; Goodwin, A. L. Nature 2015, 521, 303-309.

(29) Yokota, I. J. Phys. Soc. Jpn. 1966, 21, 420-423.

(30) Okazaki, H. J. Phys. Soc. Jpn. 1967, 23, 355-360.

(31) Hayashi, H.; Kobayashi, M.; Yokota, I. Solid State Commun. 1979, 31, 847-850.

(32) Wolf, M.L. J. Phys. C: Solid State Phys. 1984, 17, L285-L288.

(33) Castiglione, M. J.; Madden, P. A. J. Phys.: Condens. Matter 2001, 13, 9963-9983.

(34) Shimojo, F.; Aniya, M. J. Phys. Soc. Jpn. 2005, 74, 1224-1230.

(35) Aniya, M. Integr. Ferroelectrics 2010, 115, 81-94.

(36) Adelstein, N.; Wood, B. C. Chem. Mater. 2016, 28, 7218-7231.

(37) Shannon, R. D.; Prewitt, C. T. Acta Cryst. A 1976, 32, 751-767.

(38) Goff, J.P.; Hayes, W.; Hull, S.; Hutchings, M.T. J. Phys.: Condens. Matter 1991, 3, 3677-3687.

(39) Roberts, R.B.; White, G.K. J. Phys. C: Solid State Phys. 1986, 19, 7167-7172.

(40) Voss, S.; Divinski, S. V.; Imre, A. W.; Mehrer, H.; Mundy, J. N. Solid State lonics 2005, 176, 1383-1391.

(41) Sutton, A.P.; Baluffi, R.W. Interfaces in Crystalline Materials, Oxford U.P., Oxford, 1998.

(42) Egami, T.; Levashov, V.; Aga, R.; Morris, J.R. Metall. Mater. Trans. A 2008, 39(8), 1786-1790.

(43) Sayle, T.X.T.; Sayle, L.W.L.; Sayle, D.C. Phys. Chem. Chem. Phys. 2015, 17, 4441-4447.

(44) Zhang, H.; Khalkhali, M.; Qingxia, L.; Douglas, J.F. J. Chem. Phys. 2013, 138, 12 A538.

(45) Rushton, M.J.D.; Chroneos, A. Sci. Rep. 2014, 4, 6068.

(46) Burbano, M.; Marrochelli, D.; Watson, G.W. J. Electroceram. 2014, 32, 28-36.

(47) Giannici, F.; Gregori, G.; Aliotta, C.; Longo, A.; Maier, J.; Martorana, A. Chem. Mater. 2014, 26 (20), $5994-6006$.

(48) Lucid, A.K.; Keating, P.R.L.; Allen, J.P.; Watson, G.W. J. Phys. Chem. C 2016, 120, 23430-23440.

\section{Supporting information}

Videos showing the F ion mobility in the model systems, XRPD patterns, ${ }^{19} \mathrm{~F}$ NMR spectra, images of the model systems, Arrhenius plots of the conductivities for simulated and measured systems, radial distribution functions for simulated and some of the real systems, plot of the lattice parameter, of the crystallite sizes, and of $d(\mathrm{M}-\mathrm{F})$ 
prepared from the ${ }^{19} \mathrm{~F}$ MAS NMR spectra for the real systems, additional information and discussion about the transport mechanism and superionic conduction.

TOC Graphic

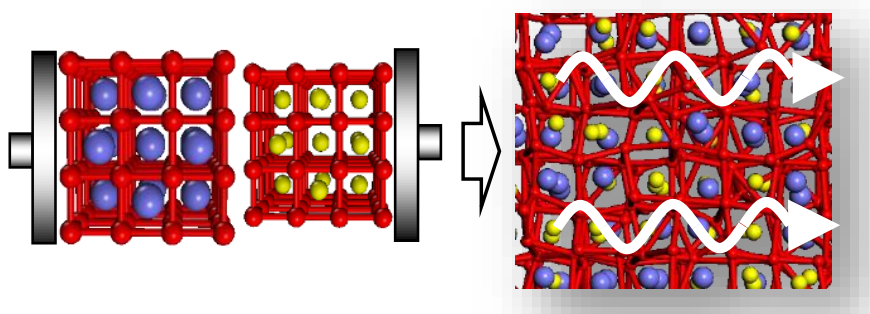

